\title{
Application of Robotic Software on Effective Diabetes Control (GH-Method: Math-Physical Medicine)
}

\author{
Hsu GC* \\ eclaireMD Foundation, USA \\ *Correspondence: Gerald C Hsu, eclaireMD Foundation, USA
}

Received on 17 June 2020; Accepted on 03 July 2020; Published on 13 July 2020

Copyright $(92020$ Hsu GC. This is an open access article and is distributed under the Creative Commons Attribution License, which permits unrestricted use, distribution, and reproduction in any medium, provided the original work is properly cited.

\begin{abstract}
This paper focuses on the author's invented robotic software technology, the artificial intelligence glucometer $(A I G)$ product, to provide a diagnosis for diabetes disease and glucose control. From 2010-2013, he self-studied internal medicine and food nutrition. In 2014, he further utilized topology concept, partial differential equation, non-linear algebra, and finite element engineering concept to develop a human metabolism's mathematical model. It consists of 10 categories and $\sim 500$ elements with $\sim 1.5$ million collected data of his own body health, disease conditions, and lifestyle details. Starting from 2015, he focused on the root cause of diabetes, which is "glucose". By applying wave theory, signal processing, energy theory, optical physics, structural \&amp; fluid dynamics from physics and engineering modeling; pattern and segmentation analysis, time/spacelfrequency domain analyses, big data analytics, machine learning and self-correction, prediction equations from mathematics and computer science, he decided to utilize his robotic software as the foundation to further build up his needed medical research and clinical tools. By using the artificial intelligence (AI) robotic software, the author's average glucose decreased from $280 \mathrm{mg} / \mathrm{dL}$ to $118 \mathrm{mg} / \mathrm{dL}$ and his hemoglobin AlC (HbAlC or AlC) reduced from 10\%+ to below 6.5\%, without diabetes medications. All his diabetes complications are either under control or have subsided. This innovative technology of his robotic software for glucose prediction and diabetes control has also been proven by many other patients, who have achieved equally remarkable medical results.
\end{abstract}

Keywords: artificial intelligence, type 2 diabetes, metabolic disorders, glucose control, diabetes

Abbreviations: AI: artificial intelligence; AIG: artificial intelligence glucometer; HbA1C or A1C: hemoglobin A1C; T2D: type 2 diabetes; FPG: fasting plasma glucose; PPG: postprandial plasma glucose 


\section{Introduction}

The author studied artificial intelligence (AI) since early 1970 and pursued his career as an engineer utilizing AI as one of his core technologies. He has been diagnosed with severe type 2 diabetes (T2D) in 1995. He suffered the most common diabetes complications including five cardiac episodes. In the beginning of 2010, he spent 25,000 $\mathrm{h}$ to selfstudy and research endocrinology and metabolic disorders, specifically diabetes. This paper focuses on his invented robotic software technology, the artificial intelligence glucometer (AIG) product, to provide a diagnosis for diabetes disease and glucose control.

\section{Methods}

Starting from 2002, he established a team with 700 software R\&D engineers to build a robotic software aiming at various business applications. In the subsequent eight years, he went through seven developmental cycles, including creation, modification, cancellation, and redevelopment. Its major modules include core database, input and output, analysis engine, display modules (tables and graphs), decision tree, self-learning, correction, and more. The user only needs to type in the system requirements or functional demands by using his/her fingers on the keyboard, then AI will automatically convert these input commands into either Java or C++ language-based software code to perform all of the follow-on necessary tasks.

By 2010, this robotic software development project was completed and ready to be commercialized; however, the author, also the technology inventor and project leader, became extremely ill due to his diabetes conditions and preparation to start medical treatment for kidney dialysis. At that moment, he had no choice but to cease the robotic software project in order to focus on saving his own life.

From 2010-2013, he self-studied internal medicine and food nutrition. In 2014, he further utilized topology concept, partial differential equation, non-linear algebra, and finite element engineering concept to develop a human metabolism's mathematical model. It consists of 10 categories and $~ 500$ elements with $\sim 1.5$ million collected data of his own body health, disease conditions, and lifestyle details. Starting from 2015, he focused on the root cause of diabetes, which is "glucose". By applying wave theory, signal processing, energy theory, optical physics, structural \& fluid dynamics from physics and engineering modeling; pattern and segmentation analysis, time/space/frequency domain analyses, big data analytics, machine learning and self-correction, prediction equations from mathematics and computer science, he decided to utilize his robotic software as the foundation to further build up his needed medical research and clinical tools.

For the past 9 years of medical research, he did not engage with any other medical associates or hire any technical assistant to help with his medical research project. His only companion (associate and assistant) is his finished robotic software from 10 years ago and medical books/papers.

\section{Results}

He embedded his medical research findings and results into his mathematical algorithms inside his robotic software. In general, medical doctors use hemoglobin $\mathrm{A} 1 \mathrm{C}$ (HbA1C or A1C) as one of the main tests to diagnose T2D. A1C contains about 20-25\% of input from fasting plasma glucose (FPG) in the morning on an empty stomach and 75-80\% of input from postprandial plasma glucose (PPG) at $2 \mathrm{~h}$ after the first bite of food. Their major influential factor contributions are weight for FPG (83\%), carbs/sugar intake (38\%), and post-meal exercise (41\%) for PPG. His developed AIG tool (Figure 1) is totally non-invasive - no need to draw the patient's blood to obtain the glucose level. His AI tool provides a range of accuracy above $90 \%$, while his own data has achieved an amazing $99.97 \%$ accuracy. This AIG tool contains a large background databank stored on the Amazon server. It contains $\sim 8$ million data from multiple sources, including the author's 5,000 personal meal photos and metadata. Not only can the AIG predict a patient's glucose level, but it will also provide immediate recommendations for both diet and exercise to the patient. AIG is the expanded version or the extended medical arm of the author's previously developed software that started 
18 years ago in 2002. By using the AI robotic software, the author's average glucose decreased from $280 \mathrm{mg} / \mathrm{dL}$ to $118 \mathrm{mg} / \mathrm{dL}$ and his A1C reduced from 10\%+ to below 6.5\% (Figure 2), without diabetes medications. All his diabetes complications are either under control or have subsided. His dedicated medical research efforts and developed AIG have indeed saved his own life!

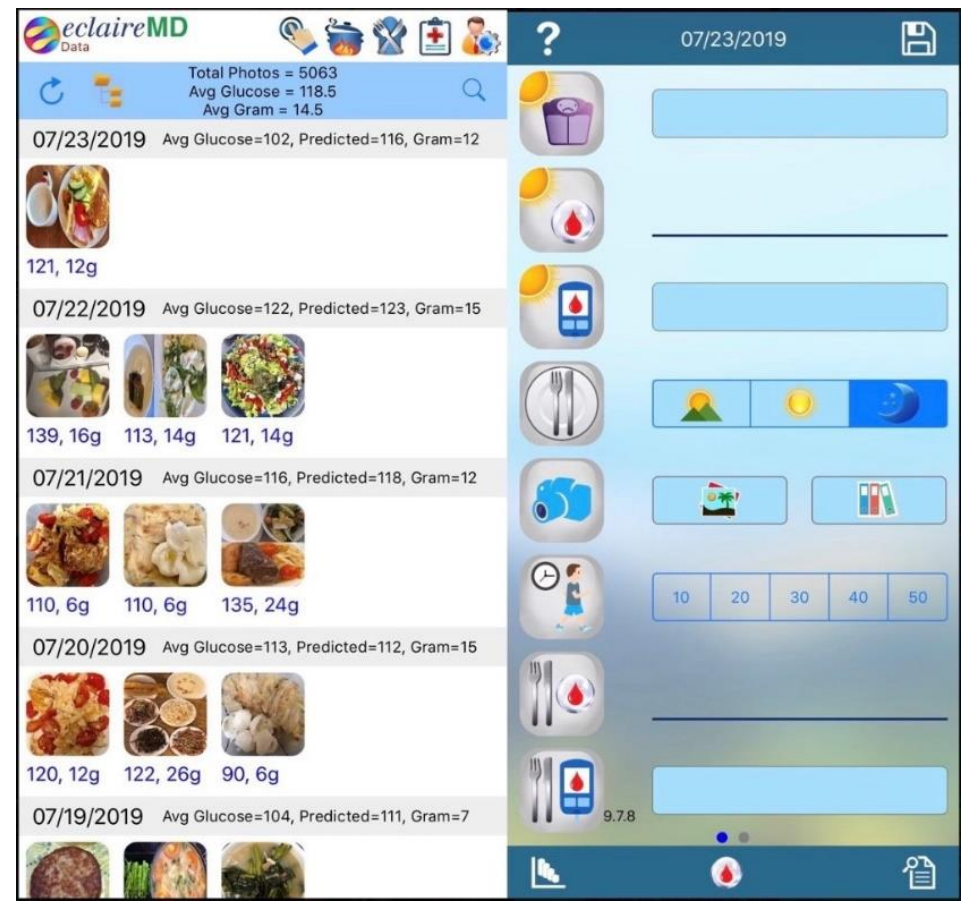

Figure 1: Artificial intelligence glucometer (AIG).

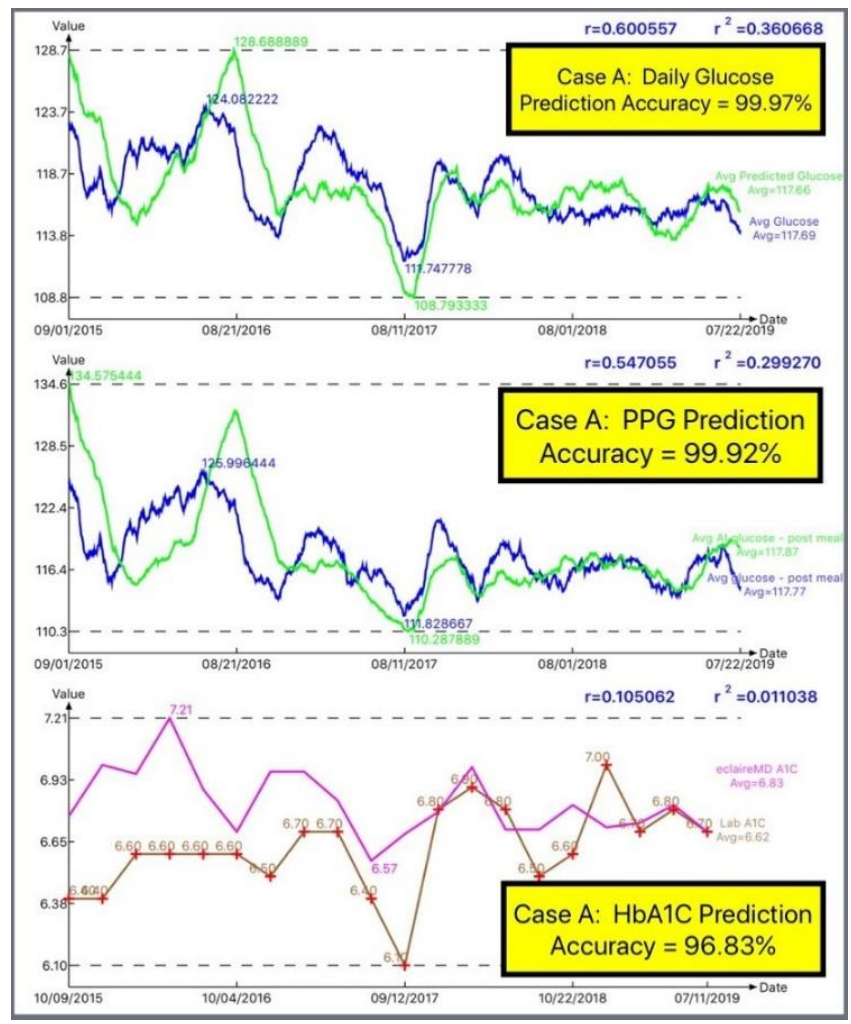

Figure 2: The author's glucose results (Case A). 
This innovative technology of his robotic software for glucose prediction and diabetes control has also been proven by many other patients, who have achieved equally remarkable medical results. Figure depicts four clinical case results with highly accurate PPG predictions and diabetes condition improvements (Figure 3). Seven clinical cases of using AIG with 95\% accuracy of PPG prediction have been listed (Figure 4).

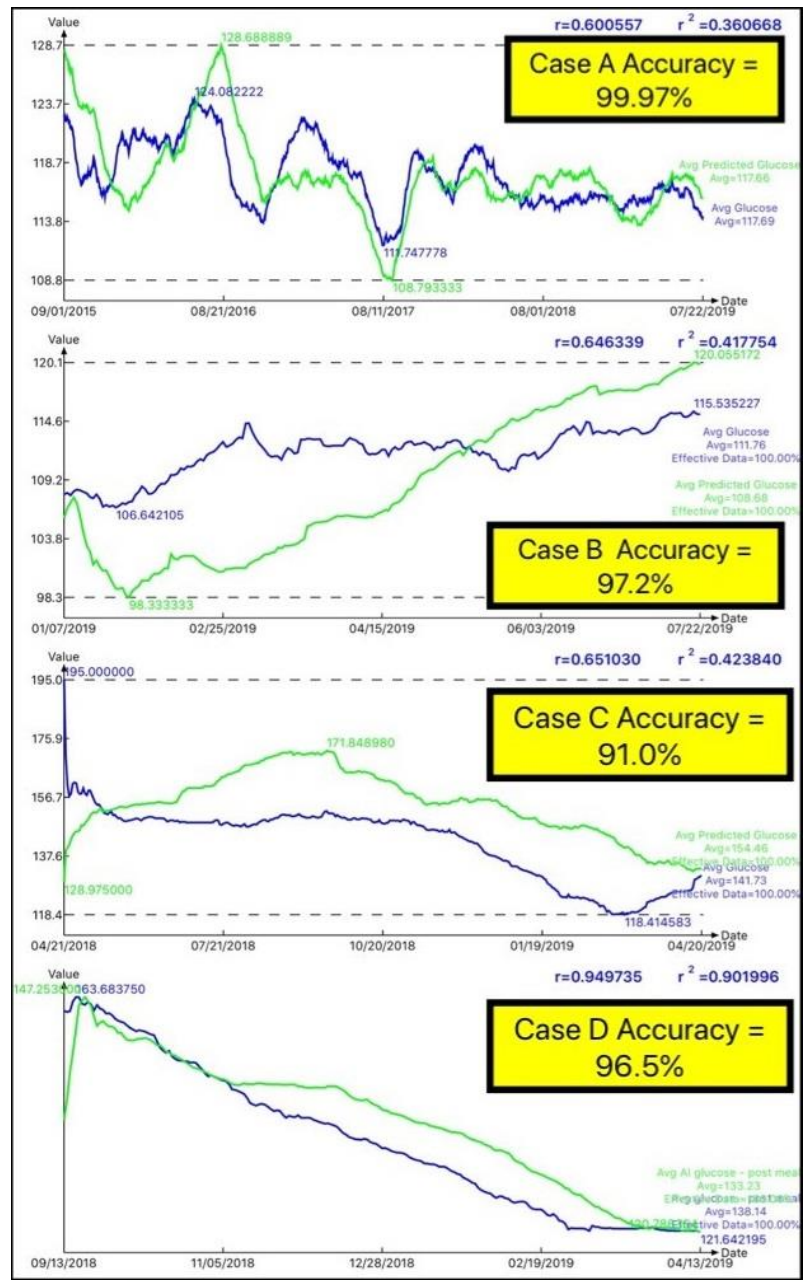

Figure 3: Four clinical cases of glucose prediction accuracies (between $91 \%$ and 100\%).

\begin{tabular}{|r|l|}
\hline Case 1 & $95 \%$ \\
\hline Case 2 & $98 \%$ \\
\hline Case 3 & $91 \%$ \\
\hline Case 4 & $91 \%$ \\
\hline Case 5 & $92 \%$ \\
\hline Case 6 & $96 \%$ \\
\hline Case 7 & $99 \%$ \\
\hline Avg. Accuray & $\mathbf{9 5 \%}$ \\
\hline
\end{tabular}

Figure 4: Artificial intelligence glucometer' accuracy of PPG prediction. 


\section{Conclusion}

Throughout the 20th century, the industrial automation has greatly changed the human productivities but also severely impacted the fate of several political empires. During this 21 st century, application domain-know-how combined with advanced AI and robotic technology will continuously revolutionize the human future in multiple aspects. The author's invention on robotic software and revolutionary medical research methodology (GH-method: math-physical medicine approach) will be extremely helpful to save many patients and improve their quality of life. 